\title{
Théorie de la diffusion pour le modèle de Nelson et problème infrarouge
}

\author{
Christian Gérard
}

\begin{abstract}
Résumé
Nous considérons dans cet exposé la théorie de la diffusion pour des modèles de Pauli-Fierz sans masse divergents infrarouge. Nous montrons que les représentations CCR obtenues a partir des champs asymptotiques contiennent des secteurs cohérents décrivant un nombre infini de bosons asymptotiquement libres. Nous formulons quelques conjectures qui conduisent a une notion bien définie de sections efficaces inclusives et non inclusives pour les Hamiltoniens de Pauli-Fierz. Finalement nous donnons une description générale de la théorie de la diffusion pour des modèles de théorie quantique des champs en présence de secteurs cohérents pour les représentations CCR asymptotiques.
\end{abstract}

\begin{abstract}
We consider in this paper the scattering theory of infrared divergent massless Pauli-Fierz Hamiltonians. We show that the CCR representations obtained from the asymptotic field contain so-called coherent sectors describing an infinite number of asymptotically free bosons. We formulate some conjectures leading to mathematically well defined notion of inclusive and non-inclusive scattering cross-sections for Pauli-Fierz Hamiltonians. Finally we give a general description of the scattering theory of QFT models in the presence of coherent sectors for the asymptotic CCR representations.
\end{abstract}

\section{Introduction}

Nous allons présenter dans cet exposé des résultats obtenus en collaboration avec Jan Dereziński.

Le problème infrarouge dans l'électrodynamique quantique, qui décrit l'interaction entre des photons et des électrons et positrons, provient de la divergence pour des moments petits de certaines intégrales utilisées pour calculer des sections efficaces de diffusion. Il est difficile d'analyser mathématiquement ce problème car l'électrodynamique quantique n'a pour le moment pas de description rigoureuse en

MSC 2000: 81T10, 81T08, 81UXX.

Keywords : théorie de la diffusion, problème infrarouge, théorie des champs. 
terme d'une dynamique unitaire sur un espace de Hilbert. Néanmoins la difficulté de la construction de l'électrodynamique quantique vient principalement des divergences ultraviolettes. En fait le problème infrarouge est aussi présent dans divers modèles simplifiés qui ont des troncatures ultraviolettes ou qui traitent soit les photons soit les électrons de manière classique.

Ces modèles peuvent être construits de manière rigoureuse et on remarque que le problème infrarouge est en fait le symptome d'une théorie de la diffusion pathologique.

\section{Le problème infrarouge dans divers modéles de théorie des champs}

\section{Mécanique quantique non relativiste}

Si on considère des électrons non-relativistes et si on traite le champ électromagnétique de manière classique dans la jauge de Coulomb, on obtient le Hamiltonien de Schrödinger du problème à $N$ corps :

$$
H=\sum_{i=1}^{N} \frac{-1}{2 m_{i}}\left(D_{x_{i}}-q_{i} A\left(x_{i}\right)\right)^{2}+\sum_{i<j} \frac{q_{i} q_{j}}{\left|x_{i}-x_{j}\right|},
$$

où $m_{i}, q_{i}$ sont les masses et charges des particules, et $A(x)$ le potentiel magnétique extérieur. L'espace de Hilbert est soit $\otimes^{N} L^{2}\left(\mathbb{R}^{3}, \mathrm{~d} x_{i}\right)$ soit $\bigwedge^{N} L^{2}\left(\mathbb{R}^{3}, \mathrm{~d} x\right)$, selon que l'on prend en compte ou non le caractère fermionique des particules.

Il est bien connu que, à cause du caractère à longue portée du potentiel de Coulomb, il n'existe pas de trajectoires asymptotiques à des trajectoires libres, et donc les opérateurs d'onde usuels n'existent pas. Par conséquent, si on utilise les méthodes usuelles pour calculer les sections efficaces, par exemple en introduisant une troncature 'adiabatique' $\mathrm{e}^{-\epsilon|t|}$ devant le potentiel d'interaction et en faisant à la fin des calculs tendre $\epsilon$ vers 0 , on obtient des quantités infinies.

La solution du problème infrarouge dans ce cas est bien connue : il faut introduire des opérateurs d'onde modifiés, par exemple à la Dollard. On obtient alors un opérateur de diffusion bien défini, mais qui n'est plus canonique (différents choix de modificateurs donnent différents opérateurs de diffusion).

\section{Modèle de charges classiques}

On traite maintenant les électrons et positrons de manière classique. En simplifiant encore le modèle en considérant des photons scalaires, le modèle est décrit par la quantification de l'équation des ondes :

$$
\square \varphi=\rho,
$$

où $\rho$ est une distribution de charge classique avec

$$
\rho(t, x) \sim \rho\left(x-v_{ \pm} t\right) \text {, quand } t \rightarrow \pm \infty \text {. }
$$


On cherche des solutions $(\phi, \pi)$ où $\pi(t, x)=\partial_{t} \phi(t, x)$ distributions à valeurs opérateurs autoadjoints, vérifiant les relations de commutation canoniques

$$
\begin{aligned}
& {\left[\phi(t, x), \phi\left(t, x^{\prime}\right)\right]=\left[\pi(t, x), \pi\left(t, x^{\prime}\right)\right]=0,} \\
& {\left[\pi(t, x), \mathrm{i} \phi\left(t, x^{\prime}\right)\right]=\delta\left(x-x^{\prime}\right) \mathbb{1} .}
\end{aligned}
$$

On fixe la condition aux limites :

$$
\lim _{t \rightarrow-\infty}(\varphi(t, x), \pi(t, x))=(\varphi(x), \pi(x))
$$

où $\varphi(x)=\phi_{F}\left(\left|D_{x}\right|^{-\frac{1}{2}} \delta_{x}\right), \pi(x)=\phi_{F}\left(\mathrm{i}\left|D_{x}\right|^{\frac{1}{2}} \delta_{x}\right)$, et $\phi_{F}($.$) désigne l'opérateur de champ$ dans la représentation de Fock, défini plus loin.

Dans ce modèle le problème infrarouge se manifeste par le fait suivant : pour tout $t<+\infty,(\varphi(t, x), \pi(t, x))$ est unitairement équivalent à $\left(\varphi_{F}(x), \pi_{F}(x)\right)$, mais si $v_{+} \neq v_{-}$

$$
(\varphi(+\infty, x), \pi(+\infty, x)):=\lim _{t \rightarrow+\infty}(\varphi(t, x), \pi(t, x))
$$

n'est pas unitairement équivalent à $\left(\varphi_{F}(x), \pi_{F}(x)\right)$.

L'interprétation est que la particule chargée crée un nombre infini de photons. (le même résultat est valable pour la quantification de l'équation de Maxwell avec une densité de courant-charge éxtérieure classique).

\section{Electrodynamique quantique}

Cette théorie est formellement décrite par la quantification des équations de Dirac-Maxwell :

$$
\left\{\begin{array}{l}
\square A_{\mu}+\frac{1}{2} \mathrm{i} e\left(\psi, \gamma_{\mu} \psi\right)=0, \\
\gamma^{\mu}\left(\partial_{\mu}-\mathrm{i} e A_{\mu}\right) \psi+m \psi=0 .
\end{array}\right.
$$

Comme nous l'avons indiqué plus haut, cette théorie n'existe pas comme modèle mathématique, principalement à cause de la divergence ultraviolette.

Le calcul formel des sections efficaces conduit à des intégrales divergentes pour $k \rightarrow 0$ (divergence infrarouge). La solution trouvée par les physiciens à ce problème est d'introduire des sections efficaces inclusives, qui consiste à négliger les photons de moment petit.

\section{Modèle de Nelson}

Le modèle de Nelson est formellement décrit par la quantification des équations de Schrödinger-ondes :

$$
\left\{\begin{array}{l}
\square \phi=\psi^{*} \psi, \\
\mathrm{i} \partial_{t} \psi+\frac{1}{2 m} \Delta_{x} \psi-(V(x)+\phi(t, x)) \psi=0,
\end{array}\right.
$$

où $(\phi, \pi)$ vérifient les CCR et $\psi, \psi^{*}$ les relations d'anticommutation canoniques :

$$
\begin{aligned}
& \left\{\psi(t, x), \psi\left(t, x^{\prime}\right)\right\}=\left\{\psi^{*}(t, x), \psi^{*}\left(t, x^{\prime}\right)\right\}=0 \\
& \left\{\psi(t, x), \mathrm{i} \psi^{*}\left(t, x^{\prime}\right)\right\}=\delta\left(x-x^{\prime}\right) \mathbb{1} .
\end{aligned}
$$


Il est facile de construire rigoureusement le modèle de Nelson avec une troncature ultraviolette. De plus Nelson a montré il y a presque 40 ans comment enlever la troncature ultraviolette, par un procédé de renormalisation assez simple. Le problème infrarouge se manifeste sur ce modèle par le fait que le hamiltonien $H$ qui décrit l'évolution temporelle n'admet pas d'état fondamental dans l'espace de Hilbert.

la théorie de la diffusion doit donc être modifiée. Dans cet exposé nous allons considérer le modèle de Nelson confiné, où l'on suppose que le potentiel éxtérieur $V$ tend vers $+\infty$ à l'infini. Dans ce cas les états de diffusion correspondent uniquement à des photons partant à l'infini, et on se concentre sur l'aspect purement photonique du problème infrarouge.

\section{Rappels sur les représentations CCR}

\subsection{Représentations CCR}

Soit $(X, \sigma)$ un espace vectoriel symplectique réel. Une représentation $C C R$ basée sur $(X, \sigma)$ est une application :

$$
X \ni h \mapsto W^{\pi}(h) \in \mathcal{U}(\mathcal{H})
$$

telle que

$$
\begin{aligned}
& W^{\pi}\left(h_{1}\right) W^{\pi}\left(h_{2}\right)=\mathrm{e}^{-\frac{\mathrm{i}}{2} \sigma\left(h_{1}, h_{2}\right)} W^{\pi}\left(h_{1}+h_{2}\right), \quad h_{1}, h_{2} \in X, \\
& W^{\pi *}(h)=W^{\pi}(-h), h \in X, \\
& W^{\pi}(0)=\mathbb{1} .
\end{aligned}
$$

Ici $\mathcal{H}$ est un espace de Hilbert et $\mathcal{U}(H)$ désigne le groupe des opérateurs unitaires sur $\mathcal{H}$.

On dit que la représentation $W^{\pi}$ est régulière si :

$$
\mathbb{R} \ni t \mapsto W^{\pi}(t h), \quad h \in X
$$

est continu pour la topologie forte de $\mathcal{B}(\mathcal{H})$.

On peut alors introduire les opérateurs de champ :

$$
\phi^{\pi}(h):=\left.\frac{\mathrm{d}}{\mathrm{id} t} W^{\pi}(t h)\right|_{t=0}, h \in X .
$$

Supposons maintenant que $(X, \sigma)$ est muni d'une structure de Kähler, c'est à dire d'une structure d'espace hermitien i, (., .) telle que $\sigma\left(h_{1}, h_{2}\right)=\operatorname{Im}\left(h_{1}, h_{2}\right)$.

On peut alors introduire les opérateurs de création et d'annihilation :

$$
a^{\pi}(h):=\frac{1}{\sqrt{2}}\left(\phi^{\pi}(h)+\mathrm{i} \phi^{\pi}(\mathrm{i} h)\right), \quad a^{\pi *}(h):=\frac{1}{\sqrt{2}}\left(\phi^{\pi}(h)-\mathrm{i} \phi^{\pi}(\mathrm{i} h)\right) .
$$




\subsection{La représentation de Fock}

soit $\mathfrak{h}$ un espace de Hilbert. On note par $\Gamma(\mathfrak{h})$ l'espace de Fock bosonique sur $\mathfrak{h}$ défini par :

$$
\Gamma(\mathfrak{h}):=\bigoplus_{n=0}^{\infty} \otimes_{\mathrm{s}}^{n} \mathfrak{h} .
$$

Le vecteur $\Omega=(1,0, \ldots$,$) est appelé vide, et l'opérateur N$ tel que $N_{\mid \otimes_{s}^{n} \mathfrak{h}}=n \mathbb{1}$ est l'opérateur de nombre. On note par

$$
S: \otimes^{n} \mathfrak{h} \rightarrow \otimes_{\mathrm{s}}^{n} \mathfrak{h}
$$

l'opérateur de symétrisation et on introduit les opérateurs de création

$$
a^{*}(h) u:=\sqrt{n+1} S u \otimes h, \text { pour } u \in \otimes_{\mathrm{s}}^{n} \mathfrak{h}, h \in \mathfrak{h},
$$

et les opérateurs d'annihilation

$$
a(h) u:=\sqrt{n}(h, u)_{\mathfrak{h}} \text { pour } u \in \otimes_{\mathrm{s}}^{n} \mathfrak{h}, h \in \mathfrak{h},
$$

qui vérifient les relations de commutation

$$
\left[a(h), a^{*}(g)\right]=(h \mid g)_{\mathfrak{h}} \mathbb{1},[a(h), a(g)]=\left[a^{*}(h), a^{*}(g)\right]=0 .
$$

On aura aussi besoin des opérateurs de champ

$$
\phi(h):=\frac{1}{\sqrt{2}}\left(a(h)+a^{*}(h)\right),
$$

qui sont essentiellement autoadjoints $\operatorname{sur} \mathcal{D}\left(N^{\frac{1}{2}}\right)$.

Les opérateurs

$$
W(h):=\mathrm{e}^{i \phi(h)}
$$

sont appelés opérateurs de Weyl, et forment une représentation CCR régulière basée $\operatorname{sur}(\mathfrak{h}, \sigma)$, pour $\sigma(h, g)=\operatorname{Im}(h, g)$.

Si $b$ est un opérateur sur $\mathfrak{h}$, on note $\mathrm{d} \Gamma(b)$ l'opérateur sur $\Gamma(\mathfrak{h})$ défini par :

$$
\left.\mathrm{d} \Gamma(b)\right|_{\bigotimes_{\mathrm{s}}^{n} \mathfrak{h}}:=\sum_{j=1}^{n} \mathbb{1}^{\otimes(j-1)} \otimes b \otimes \mathbb{1}^{\otimes(n-j)}
$$

Finalement si $\mathfrak{h}=L^{2}\left(\mathbb{R}^{d}, \mathrm{~d} k\right)$ on introduit les expressions $a(k), a^{*}(k)$ définies par

$$
a(f)=: \int a(k) \bar{f}(k) d k, a^{*}(f)=: \int a^{*}(k) f(k) d k .
$$

Si $h \in \mathfrak{h}, W(-\mathrm{i} h) \Omega$ est appelé le vecteur cohérent centré en $h$. Il vérifie les identités :

$$
a(f) W(-\mathrm{i} h) \Omega=\frac{1}{\sqrt{2}}(f \mid h) W(-\mathrm{i} h) \Omega .
$$

Cette propriété caractérise les vecteurs cohérents. 


\subsection{Représentations cohérentes}

Soit maintenant $\mathfrak{h}_{0} \subset \mathfrak{h}$ un sous espace vectoriel dense. On note par $\mathfrak{h}_{0}^{*}$ l'espace des formes antilinéaires sur $\mathfrak{h}_{0}$ et on note par $<., .>$ le crochet de dualité entre $\mathfrak{h}_{0}$ et $\mathfrak{h}_{0}^{*}$. Le produit scalaire de $\mathfrak{h}$ permet d'injecter canoniquement $\mathfrak{h}$ dans $\mathfrak{h}_{0}^{*}$.

L'application

$$
\mathfrak{h}_{0} \ni h \mapsto W^{g}(h):=W(h) \mathrm{e}^{\mathrm{i} R e<g, h>} \in U(\Gamma(\mathfrak{h}))
$$

est une représentation CCR régulière appelée représentation $g-$ cohérente.

Les opérateurs de champ, création et annihilation associés seront notés $\phi^{g}(h)$, $a^{g}(h)$ et $a^{g *}(h)$.

On a :

$$
\begin{aligned}
& \phi^{g}(h)=\phi(h)+\operatorname{Re}<g, h>, \\
& a^{g *}(h)=a^{*}(h)+\frac{1}{\sqrt{2}}<g, h>, \\
& a^{g}(h)=a(h)+\frac{1}{\sqrt{2}} \overline{<g, h>.}
\end{aligned}
$$

Il est facile de voir que la représentation $W^{g}($.$) est unitairement équivalente à la$ représentation de Fock $W($.$) si et seulement si g \in \mathfrak{h}$, et on a alors :

$$
W^{g}(h)=W(\mathrm{i} g) W(h) W(-\mathrm{i} g), h \in \mathfrak{h} .
$$

\section{Le modèle de Nelson confiné}

Nous décrivons d'abord rapidement le hamiltonien de Nelson.

L'électron est décrit par l'espace de Hilbert $\mathcal{K}:=L^{2}\left(\mathbb{R}^{3}, \mathrm{~d} x\right)$ et par l'hamiltonien $K=\frac{1}{2} D_{x}^{2}+V(x)$, où $V$ est un potentiel réel. On supposera dans la suite que

$$
\lim _{x \rightarrow \infty} V(x)=+\infty
$$

c'est à dire que l'électron est confiné.

Le champ de photons scalaires est décrit par l'espace à une particule $\mathfrak{h}=$ $L^{2}\left(\mathbb{R}^{3}, \mathrm{~d} k\right)$, où $k$ est le moment d'un photon, et par la relation de dispersion $\omega(k)=$ $|k|$.

Le champ quantique de photons est alors décrit par l'espace de Hilbert $\Gamma(\mathfrak{h})$ et le hamiltonien $\mathrm{d} \Gamma(\omega)$.

Le système en interaction est décrit par l'espace de Hilbert $\mathcal{H}=\mathcal{K} \otimes \Gamma(\mathfrak{h})$ et le hamiltonien libre

$$
H_{0}=K \otimes \mathbb{1}+\mathbb{1} \otimes \mathrm{d} \Gamma(\omega) .
$$

L'interaction est décrite à l'aide d'une fonction :

$$
\mathbb{R}^{3} \ni k \mapsto v(k)=|k|^{-\frac{1}{2}} \chi(|k| \leq \Lambda) \mathrm{e}^{-\mathrm{i} k \cdot x} \in B(\mathcal{K}) .
$$

Ici la fonction $\chi$ est une troncature ultraviolette, qui tronque l'interaction à des énergies supérieures à $\Lambda$. Il est possible d'enlever cette troncature en définissant 
un hamiltonien renormalisé. (Le traitement du problème infrarouge pour le modèle renormalisé ne devrait sans doute présenter que quelques difficultés techniques supplémentaires). On pose alors

$$
V:=\frac{1}{\sqrt{2}} \int v(k) \otimes a^{*}(k)+v^{*}(k) \otimes a(k) \mathrm{d} k,
$$

et le hamiltonien de Nelson est :

$$
H:=H_{0}+V
$$

Lemma 4.1 Le hamiltonien $H$ est autoadjoint et borné inférieurement sur $\mathcal{D}\left(H_{0}\right)$ et on a $\sigma(H)=[\inf \sigma(H),+\infty[$.

\section{Théorie de la diffusion pour le modèle de Nelson}

La théorie de la diffusion du modèle de Nelson est basée sur le formalisme LSZ (pour Lehmann-Szymanzik-Zimmermann), et commence par la construction des champs asymptotiques. Pour des modéles non confinés, il faudrait utiliser à la place une version du formalisme de Haag-Ruelle.

\subsection{Représentations asymptotiques}

Soit $\mathfrak{h}_{0}=\mathcal{D}\left(\omega^{-\frac{1}{2}}\right)$ et $h_{t}=\mathrm{e}^{-\mathrm{i} t \omega} h$, pour $h \in \mathfrak{h}_{0}$.

Theorem 5.1 (i) $\forall h \in \mathfrak{h}_{0}$ les opérateurs de Weyl asymptotiques

$$
W^{ \pm}(h):=\mathrm{s}-\lim _{t \rightarrow \pm \infty} \mathrm{e}^{\mathrm{i} t H} W\left(h_{t}\right) \mathrm{e}^{-\mathrm{i} t H} \text { existent. }
$$

(ii) les applications $\mathfrak{h}_{0} \ni h \mapsto W^{ \pm}(h)$ sont des représentations $C C R$ régulières. (iii) on a la propriété d'entrelacement : $\mathrm{e}^{\mathrm{i} t H} W^{ \pm}(h) \mathrm{e}^{-\mathrm{i} t H}=W^{ \pm}\left(h_{-t}\right), t \in \mathbb{R}$.

En appliquant le formalisme général, on obtient les opérateurs de champ asymptotiques :

$$
\phi^{ \pm}(h):=\mathrm{i}^{-1} \frac{\mathrm{d}}{\mathrm{d} t} W^{ \pm}(t h)_{\mid t=0}, h \in \mathfrak{h}_{0},
$$

et les créateurs-annihilateurs asymptotiques:

$$
\begin{aligned}
& a^{ \pm}(h):=\frac{1}{\sqrt{2}}\left(\phi^{ \pm}(h)+\mathrm{i} \phi^{ \pm}(\mathrm{i} h)\right), \\
& a^{ \pm *}(h):=\frac{1}{\sqrt{2}}\left(\phi^{ \pm}(h)-\mathrm{i} \phi^{ \pm}(\mathrm{i} h)\right) .
\end{aligned}
$$

L'étude de la théorie de la diffusion revient alors à analyser la nature des représentations asymptotiques $W^{ \pm}$. En particulier on peut se demander si elles sont unitairement équivalentes à une représentation de Fock, ou à une représentation cohérente. 


\subsection{Hamiltonien renormalisé et opérateurs d'entrelacement}

Dans l'étude des représentations asymptotiques, on est conduit à considérer un hamiltonien auxilliaire appelé hamiltonien renormalisé, dont nous donnons maintenant la définition.

Soit $g(k)=-\mathrm{i} \frac{\rho(k)}{\omega(k)}$, pour $\rho(k)=|k|^{-\frac{1}{2}} \chi(|k| \leq \Lambda), g_{\sigma}=g \mathbb{1}_{\{\omega \geq \sigma\}}, 0<\sigma \leq 1$. On remarque que $g \in \mathfrak{h}_{0}^{*}$, mais $g \notin \mathfrak{h}$. On montre facilement que :

$$
\lim _{\sigma \rightarrow 0} \mathrm{e}^{\mathrm{i} \phi\left(g_{\sigma}\right)}(z-H)^{-1} \mathrm{e}^{-\mathrm{i} \phi\left(g_{\sigma}\right)}=:\left(z-H_{\text {ren }}\right)^{-1} \text { existe, } z \in \mathbb{C} \backslash \mathbb{R} .
$$

$H_{\text {ren }}$ est le hamiltonien renormalisé. On a :

$$
H_{\mathrm{ren}}=K_{1} \otimes \mathbb{1}+\mathbb{1} \otimes \mathrm{d} \Gamma(\omega)+\phi\left(v_{1}\right),
$$

pour $v_{1}(k)=\rho(k)\left(\mathrm{e}^{-i k \cdot \mathrm{x}}-1\right), K_{1}=K-\operatorname{Re}\left(v_{0}, \omega^{-1} v_{1}\right)_{\mathfrak{h}}+\frac{1}{2} \operatorname{Re}\left(v_{0}, \omega^{-1} v_{0}\right)_{\mathfrak{h}}$.

Comme $g \notin \mathfrak{h}$ on a w- $\lim _{\sigma \rightarrow 0} \mathrm{e}^{\mathrm{i} \phi\left(g_{\sigma}\right)}=0$. En language imagé, ceci veut dire que la translation de phase de vecteur $g$ 'sort' de l'espace symplectique. En particulier $H$ et $H_{\text {ren }}$ ne sont pas unitairement équivalents. Par exemple $\sigma\left(H_{\text {ren }}\right)=\sigma(H)$, inf $\sigma(H)$ est une valeur propre de $H_{\text {ren }}$, inf $\sigma(H)$ n'est pas une valeur propre de $H$.

Une autre remarque est que les opérateurs de Weyl asymptotiques pour $H_{\text {ren }}$ existent et forment une représentation CCR régulière, par exactement les mêmes arguments que ceux utilisés pour $H$.

Posons maintenant $g_{t}=\mathrm{e}^{-\mathrm{i} t \omega} g$. On voit que $g_{t}-g \in \mathfrak{h}$ et $\operatorname{Im}\left(g_{t}, g\right)<\infty$.

Proposition 5.2 Soit $U(t)=\mathrm{e}^{\mathrm{i} t H} \mathrm{e}^{\mathrm{i} \phi\left(g_{t}-g\right)} \mathrm{e}^{-\mathrm{i} t H_{\mathrm{ren}}}$. Alors :

(i) $\mathrm{s}-\lim _{t \rightarrow \pm \infty} U(t)=: U^{ \pm}$existent,

(ii) $\mathrm{s}-\lim _{t \rightarrow \pm \infty} U^{*}(t)=U^{ \pm *}$.

Les opérateurs $U^{ \pm}$sont appelés opérateurs d'entrelacement.

Theorem 5.3 Soit $W_{\text {ren }}^{ \pm}($.$) les opérateurs de Weyl asymptotiques pour H_{\text {ren }}$. Alors :

(i) $W^{ \pm}(h) U^{ \pm}=U^{ \pm} W_{\text {ren }}^{ \pm}(h) \mathrm{e}^{-\mathrm{i} \operatorname{Im}(h, g)}, h \in \mathfrak{h}_{0}$,

(ii) $\mathrm{e}^{-\mathrm{i} t H} U^{ \pm}=U^{ \pm} W_{\text {ren }}^{ \pm}\left(g_{t}-g\right) \mathrm{e}^{-\mathrm{iIm}\left(g_{t}, g\right)} \mathrm{e}^{-\mathrm{i} t H_{\mathrm{ren}}}$.

On voit dont que les représentations asymptotiques pour $H$ sont unitairement équivalentes aux représentations asymptotiques pour $H_{\text {ren }}$ après une translation de phase par $g$.

\subsection{Opérateurs d'onde}

L'opérateur $H_{\text {ren }}$ possède un état fondamental dans l'espace de Hilbert. On s'attend donc à ce que sa théorie de la diffusion soit celle rencontrée en l'absence de problème infrarouge, comme par exemple pour des photons massifs.

Opérateurs d'onde pour $H_{\text {ren }}$

Soit

$$
\mathcal{K}_{\text {ren }}^{ \pm}=\left\{u \in \mathcal{H} \mid a_{\text {ren }}^{ \pm}(h) u=0, \forall h \in \mathfrak{h}_{0}\right\} .
$$

$\mathcal{K}_{\text {ren }}^{ \pm}$est l'espace des vides pour $W_{\text {ren }}^{ \pm}($.$) . Intuitivement il s'agit des états ne conte-$ nant aucun photon asymptotiquement libre. Il est facile de vérifier les faits suivants : 
$-\mathcal{K}_{\text {ren }}^{ \pm}$sont invariants par $\mathrm{e}^{-\mathrm{i} t H_{\text {ren }}}$ $-\mathcal{H}_{\mathrm{pp}}\left(H_{\text {ren }}\right) \subset \mathcal{K}_{\text {ren }}^{ \pm}$.

Comme $H_{\text {ren }}$ a un état fondamental, les espaces $\mathcal{K}_{\text {ren }}^{ \pm}$sont non triviaux.

On définit alors les opérateurs d'onde pour $H_{\text {ren }}$ par :

$$
\Omega_{\mathrm{ren}}^{ \pm}: \begin{aligned}
& \mathcal{K}_{\text {ren }}^{ \pm} \otimes \Gamma(\mathfrak{h}) \rightarrow \mathcal{H} \\
& \psi \otimes \Pi_{1}^{n} a^{*}\left(h_{i}\right) \Omega \mapsto \Pi_{1}^{n} a^{ \pm *}\left(h_{i}\right) \psi, h_{i} \in \mathfrak{h}_{0} .
\end{aligned}
$$

Proposition 5.4 i) $\Omega_{\mathrm{ren}}^{ \pm}$sont isométriques,

ii) $\Omega_{\text {ren }}^{ \pm} \mathbb{1}_{\mathcal{K}_{\text {ren }}^{ \pm}} \otimes W(h)=W_{\text {ren }}^{ \pm}(h) \Omega_{\text {ren }}^{ \pm}, h \in \mathfrak{h}_{0}$,

iii) $\mathrm{e}^{-\mathrm{i} t H_{\text {ren }}} \Omega_{\text {ren }}^{ \pm}=\Omega_{\text {ren }}^{ \pm} \mathrm{e}^{-\mathrm{i} t H_{\text {ren }}^{ \pm}}$, où $H_{\text {ren }}^{ \pm}$sont les hamiltoniens asymptotiques :

$$
H_{\text {ren }}^{ \pm}=K_{\text {ren }}^{ \pm} \otimes \mathbb{1}+\mathbb{1} \otimes \mathrm{d} \Gamma(\omega), K_{\text {ren }}^{ \pm}:=H_{\text {ren } \mid \mathcal{K}_{\text {ren }}^{ \pm}} .
$$

Soit $\mathcal{H}_{\mathrm{ren}}^{ \pm}:=\operatorname{Im} \Omega_{\text {ren }}^{ \pm}$. Alors :

iv) $\mathcal{H}_{\mathrm{ren}}^{ \pm}$sont invariants par $\mathrm{e}^{-\mathrm{i} t H_{\mathrm{ren}}}$ et $W_{\mathrm{ren}}^{ \pm}(h)$,

v) les représentations $C C R W_{\mathrm{ren}}^{ \pm}($.$) restreintes à \mathcal{H}_{\mathrm{ren}}^{ \pm}$sont unitairement équivalentes à des représentations de Fock.

\section{Opérateurs d'onde pour $H$}

Soit maintenant

$$
\mathcal{K}^{ \pm}:=U^{ \pm} \mathcal{K}_{\text {ren }}^{ \pm}=\left\{u \in \mathcal{H} \mid a^{ \pm}(h) u=\frac{\mathrm{i}}{\sqrt{2}}(h, g) u, \forall h \in \mathfrak{h}_{0}\right\}
$$

L'espace $\mathcal{K}^{ \pm}$est donc l' espace des vecteurs $g$-cohérents pour $W^{ \pm}($.$) .$

On pose aussi $\mathcal{H}^{ \pm}:=U^{ \pm} \mathcal{H}_{\text {ren }}^{ \pm}$. Les espaces $\mathcal{H}^{ \pm}$sont invariants par e $\mathrm{e}^{-\mathrm{i} t H}$ et $W^{ \pm}($.$) ,$ $h \in \mathfrak{h}_{0}$.

Les opérateurs d'onde pour $H$ sont définis par :

$$
\begin{array}{ll}
\Omega^{ \pm}: & \mathcal{K}^{ \pm} \otimes \Gamma(\mathfrak{h}) \rightarrow \mathcal{H} \\
& \Omega^{ \pm}:=U^{ \pm} \Omega_{\text {ren }}^{ \pm} U^{ \pm *} \otimes \mathbb{1}
\end{array}
$$

Proposition 5.5 i) $\Omega^{ \pm} \mathbb{1}_{\mathcal{K}^{ \pm}} \otimes W(h)=W^{ \pm}(h) \mathrm{e}^{\mathrm{i} \operatorname{Im}(h, g)} \Omega^{ \pm}, h \in \mathfrak{h}_{0}$,

ii) $\mathrm{e}^{-\mathrm{i} t H} \Omega_{\mathrm{ren}}^{ \pm}=\Omega_{\mathrm{ren}}^{ \pm} \mathrm{e}^{-\mathrm{i} t H^{ \pm}}$, pour

$$
\begin{gathered}
H^{ \pm}=K^{ \pm} \otimes \mathbb{1}+\mathbb{1} \otimes \mathrm{d} \Gamma_{g}(\omega), K^{ \pm}:=U^{ \pm} K_{\text {ren }}^{ \pm} U^{ \pm *}, \\
\mathrm{~d} \Gamma_{g}(\omega):=\int \omega(k)\left(a^{*}(k)-\frac{\mathrm{i}}{\sqrt{2}} \bar{g}(k)\right)\left(a(k)+\frac{\mathrm{i}}{\sqrt{2}} g(k)\right) \mathrm{d} k,
\end{gathered}
$$

iii) les représentations $W^{ \pm}($.$) restreints à \mathcal{H}^{ \pm}$sont des représentations CCR $g$-cohérentes.

Le hamiltonien $\mathrm{d} \Gamma_{g}(\omega)$ est appelé en physique hamiltonien de van Hove. 


\subsection{Propriétés de covariance}

On remarque que la définition des opérateurs d'onde dépend du choix du facteur cohérent $g$. En fait on pourrait retrancher à $g$ un élément de $L^{2}\left(\mathbb{R}^{3}, \mathrm{~d} k\right.$ ) (par exemple en tronquant $g$ dans $\{|k| \leq \epsilon\}$ ) et obtenir d'autres opérateurs d'onde. Ce caractère non canonique des opérateurs d'onde n'est pas surprenant, on le rencontre aussi dans le problème de Coulomb, comme on l'a indiqué dans l'introduction. On est donc conduit naturellement à étudier la covariance des différents objets introduits plus haut par un changement $g \rightarrow \tilde{g}=g+f$, pour $f \in \mathfrak{h}$.

\section{Covariance des objets renormalisés :}

$$
\begin{aligned}
& \tilde{H}_{\text {ren }}=\mathrm{e}^{\mathrm{i} \phi(f)} H_{r e n} \mathrm{e}^{-\mathrm{i} \phi(f)}, \\
& \tilde{W}_{\text {ren }}^{ \pm}(h)=\mathrm{e}^{\mathrm{i} \phi(f)} W_{\text {ren }}^{ \pm}(h) \mathrm{e}^{-\mathrm{i} \phi(f)}, f \in \mathfrak{h}_{0} .
\end{aligned}
$$

On voit donc que les objets renormalisés changent de la manière évidente par le changement $g \mapsto \tilde{g}$.

\section{Covariance des objets de diffusion :}

Supposons que $f \in \mathfrak{h}_{0}$. Alors:

i) $\tilde{U}^{ \pm}=W^{ \pm}(f) U^{ \pm} \mathrm{e}^{-\mathrm{i} \phi(f)}$,

ii) $\tilde{\mathcal{K}}^{ \pm}=W^{ \pm}(f) \mathcal{K}^{ \pm}$

iii) $\tilde{\mathcal{H}}^{ \pm}=\mathcal{H}^{ \pm}$

iv) $\tilde{\Omega}^{ \pm}=W^{ \pm}(f) \Omega^{ \pm}\left(W^{ \pm}(f)^{*} \otimes \mathbb{1}\right)$.

La propriété iv) illustre bien le rôle du facteur cohérent $g$, que l'on peut, au prix d'une certaine précision mathématique, expliquer heuristiquement de la manière suivante :

un état évoluant par le hamiltonien $H$ va continuellement émettre des photons, d'énergie de plus en plus petite. Ce processus ne s'arréte pas après un nombre fini d'émissions, car le système atome+photons ne possède pas d'état fondamental dans lequel il pourrait atteindre enfin le repos. La description de la théorie de la diffusion en termes de l'émission d'un nombre fini de photons de diffusion n'est donc pas appropriée.

Le facteur cohérent $g$ représente une certaine distribution moyenne de photons, et c'est par rapport à cette distribution moyenne et non plus par rapport au vide que l'on définit les photons de diffusion. Comme le montre la propriété iv), changer $g$ revient à changer notre définition de photons de diffusion.

\section{Sections efficaces inclusives}

Le but de cette section est d'introduire une notion de section efficace inclusive pour le modèle de Nelson. Cette notion est introduite par les physiciens en électrodynamique quantique, pour obtenir des sections efficaces finies. Heuristiquement celà correspond à considérer uniquement comme photons de diffusion les photons durs de moment plus grand qu'un certain paramètre $\epsilon>0$, les photons mous étant traités avec les électrons-positrons.

Rappelons rapidement la définition des sections efficaces en Mécanique Quantique. 
Soit $\rho$ une matrice densité $(\rho \geq 0, \operatorname{Tr} \rho=1)$ décrivant l'état initial, $A$ une observable, $S$ l'opérateur de diffusion. Alors la quantité $\operatorname{Tr}\left(\rho S^{*} A S\right)$ représente la valeur moyenne de la mesure de l'obervable $A$ au temps $t=+\infty$ pour l'état initial $\rho$ au temps $t=-\infty$.

\subsection{Photons durs et photons mous}

Soit $\epsilon>0$ une échelle d'énergie. Soit $\mathfrak{h}_{\leq \epsilon}=L^{2}(\{\omega(k) \leq \epsilon\}, \mathrm{d} k), \mathfrak{h}_{>\epsilon}:=$ $L^{2}(\{\omega(k)>\epsilon\}, \mathrm{d} k)$.

On suppose que $g=\mathbb{1}_{\{\omega \leq \epsilon\}} g$, ce que l'on peut réaliser en soustrayant à $g$ une fonction dans $L^{2}\left(\mathbb{R}^{3}, \mathrm{~d} k\right)$.

On rappelle aussi la loi exponentielle pour les espaces de Fock: si $\mathfrak{h}_{1}, \mathfrak{h}_{2}$ sont deux espaces de Hilbert, il existe un opérateur unitaire $U: \Gamma\left(\mathfrak{h}_{1} \oplus \mathfrak{h}_{2}\right) \rightarrow \Gamma\left(\mathfrak{h}_{1}\right) \otimes \Gamma\left(\mathfrak{h}_{2}\right)$ tel que :

$$
\begin{aligned}
& U \Omega=\Omega \otimes \Omega, \\
& U a^{\sharp}\left(h_{1} \oplus h_{2}\right)=\left(a^{\sharp}\left(h_{1}\right) \otimes \mathbb{1}+\mathbb{1} \otimes a^{\sharp}\left(h_{2}\right) U .\right.
\end{aligned}
$$

On peut donc identifier $\Gamma(\mathfrak{h})$ avec $\Gamma\left(\mathfrak{h}_{\leq \epsilon}\right) \otimes \Gamma\left(\mathfrak{h}_{>\epsilon}\right)$. On pose alors $\mathcal{H}_{\leq \epsilon}:=\mathcal{K}^{ \pm} \otimes \Gamma\left(\mathfrak{h}_{\leq \epsilon}\right)$, $H_{\leq \epsilon}^{ \pm}:=H_{\mathcal{H}_{\leq \epsilon}^{ \pm}}^{ \pm}$, qui sont respectivement l'espace et le hamiltonien de l' atome habillé' et des photons mous. L'espace $\Gamma\left(\mathfrak{h}_{>\epsilon}\right)$ est donc l'espace des photons durs.

Par la loi exponentielle, on peut donc identifier les espaces asymptotiques $\mathcal{K}^{ \pm} \otimes$ $\Gamma(\mathfrak{h})$ avec $\mathcal{H}_{\leq \epsilon}^{ \pm} \otimes \Gamma\left(\mathfrak{h}_{>\epsilon}\right)$.

\section{Observables dans le futur}

Les observables que l'on mesure dans le futur seront de la forme $B=\mathbb{1}_{\mathcal{H}_{\leq \epsilon}^{ \pm}} \otimes A$, $A \in B\left(\Gamma\left(\mathfrak{h}_{>\epsilon}\right)\right)$. Ceci correspond à ne pas faire de mesure sur les photons mous ou sur l'atome habillé'. Notons que pour mesurer des photons d'énergie arbitrairement petite, il faudrait disposer d'un détecteur arbitrairement grand.

\section{Etats initiaux dans le passé}

Pour la même raison 'état initial du système dans le passé ne peut pas être entièrement préparé, mais on s'attend à ne pouvoir préparer que les photons durs. La classe des états initiaux admissibles est décrite de la manière suivante :

Soit $\rho_{>\epsilon}$ une matrice densité sur $\Gamma\left(\mathfrak{h}_{>\epsilon}\right)$. Les états initiaux admissibles sont les matrices densité $\rho$ sur $\mathcal{K}^{-} \otimes \Gamma(\mathfrak{h})$ telles que :

i) $\operatorname{Tr}_{\mathcal{H}_{\leq \epsilon}^{ \pm}} \rho=\rho_{>\epsilon}$, où $\operatorname{Tr}_{\mathcal{H}_{\leq \epsilon}^{ \pm}}$désigne la trace partielle par rapport à $\mathcal{H}_{\leq \epsilon}^{ \pm}$.

ii) $\rho=\mathbb{1}_{[E, E+\delta]}\left(H^{-} \rho\right), 0<\delta \leq 1$.

On pose alors :

$$
\left.\left.\operatorname{Cross}_{\delta}\left(\rho_{>\epsilon}, A\right):=\left\{\operatorname{Tr}\left(\rho S^{*}(\mathbb{1} \otimes A) S\right), \rho \text { vérifie } i\right) i i\right)\right\} .
$$

A cause de l'indétermination de l'état initial, la section efficace est maintenant un ensemble de résultats pour des états initiaux admissibles.

On vérifie facilement les propriétés suivantes :

- $\operatorname{Cross}_{\delta}\left(\rho_{>\epsilon}, A\right)$ est un intervalle, décroissant quand $\delta \rightarrow 0$,

- $\operatorname{Cross}_{\delta}\left(\rho_{>\epsilon}, A\right)$ est indépendant de $g$ modulo des éléments de $\mathfrak{h}_{\leq \epsilon}$. 
La conjecture suivante implique l'existence des sections efficaces inclusives, dans un cadre non perturbatif.

Conjecture (existence des sections efficaces inclusives)

$$
\bigcap_{0<\delta \leq 1} \operatorname{Cross}_{\delta}\left(\rho_{>\epsilon}, A\right)=\{\lambda\}
$$

\section{Références}

[Ar] Arai, A. : Ground state of massless Nelson model without infrared cutoff in a non-Fock representation, Rev. Math. Phys. 13 (2001) 1075-1094

[BSZ] Baez, J.C., Segal, I.E., Zhou, Z. : Introduction to algebraic and constructive quantum field theory, Princeton NJ, Princeton University Press (1991).

[BN] Bloch, F., Nordsieck, A. : Note on the radiation field of the electron, Phys. Rev. 52 (1937) 54-59

[BR] Bratteli, O, Robinson D. W. : Operator Algebras and Quantum Statistical Mechanics Vols I, II, Springer Berlin 1981

[De1] Dereziński, J. : Van Hove Hamiltonians - exactly solvable models of the infrared an ultraviolet problem, preprint, Maphysto 2002

[DG1] Dereziński, J., Gérard, C. : Asymptotic completeness in quantum field theory.

Massive Pauli-Fierz Hamiltonians, Rev. Math. Phys. 11 (1999)

[DG2] Dereziński, J., Gérard, C. : Spectral and scattering theory of spatially cutoff $P(\varphi)_{2}$ Hamiltonians, Comm. Math. Phys. 213 (2000) 39-125 383-450.

[F] Fröhlich, J. : On the infrared problem in a model of scalar electrons and massless scalar bosons, Ann. Inst. H. P. 19 (1973) 1-103.

[Ge1] Gérard, C. : On the existence of ground states for massless Pauli-Fierz Hamiltonians. Ann. Henri Poincaré 1 (2000), no. 3, p 443-45.

[HHS] Hirokawa, M.., Hiroshima, F. and Spohn, H. : Ground state for point particles interacting through a massless scalar Bose field, preprint 2002 (give reference)

[HK] Hoegh-Krohn, R. Boson fields under a general class of cut-off interactions, Comm. Math. Phys. 12 (1969) 216-225

[JR] Jauch, J. M., Röhrlich, F. The theory of photons and electrons, 2nd edition, Springer 1976

[Ki] Kibble, T. W. B. : Coherent soft-photon states and infrared divergences I J. Math. Phys. 9 (1968) 315 ; II Mass-shell singularities of Green's functions, Phys. Rev. 173 (1968) 1527-1535; III Asymptotic states and reduction formulas, Phys. Rev. 174 (1968) 1882-1901 ; IV The scattering, Phys. Rev. 175 (1968) 1624-1640

[KF] Kulish, P. P., Faddeev, L. D. : give title Theor. Math. Phys. 4 (1970) 745 
[LMS] Lorinczi, J., Minlos, R. Spohn, H. : The infrared behavior in Nelson's model of a quantum particle coupled to a massless scalar field, preprint 2001.

[Ne] Nelson. E : Interaction of non-relativistic particles with a quantized scalar field, J. Math. Phys. 5(1964) 1190-1197

[Pi] Pizzo A. : One particle (improper) states and scattering states in Nelson's massless model, 2000 preprint, math-ph/0010043

[Sch] Schweber, S. S. : Introduction to non-relativistic quantum field theory, Harper and Row 1962

[YFS] Yennie, D., Frautschi, S. and Suura, H. : The infrared divergence phenomena and high-energy processes Ann. Phys., 13 (1961) 379-452

DÉPARTEMENT DE MATHÉMATIQUES

UNIVERSITÉ DE PARIS SUD

91405 Orsay Cedex France

christian.gerard@math.u-psud.fr 